\section{Penzberg \\ Biotechnologiezentrum erweitert}

Mit der Eröffnung eines neuen Komplexes hat Roche in Penzberg seine globalen Produktionskapazitäten im Bereich der Diagnostik erheblich erweitert. Für den Diagnostics Operations Complex (DOC) hat der Schweizer Konzern 136 Millionen Euro investiert.

„Wir setzen klar auf den Forschungs- und Produktionsstandort Deutschland“, sagte Franz Humer, Verwaltungsratspräsident von Roche, bei der Einweihung des Neubaus. Dank der Investitionen durch Roche in den vergangenen zwölf Jahren habe sich Penzberg zum größten Biotechnologie-Zentrum in Europa entwickelt. Zugleich warnte Humer unter Hinweis auf Zwangsrabatte und die Entwicklung in der Gesundheitspolitik jedoch vor einer ,relativen Verschlechterung der Rahmenbedingungen für Innovationen" in Deutschland. Um Forschung und Innovation zu stärken, bot der Vorstand der Roche Pharma AG Deutschland, Hagen Pfund- ner, der Politik einen „Pakt für Innovation und Wertschöpfung mit der Deutschen Forschenden Pharmaindustrie" an. Der Zwangsrabatt sollte dabei auf neue Investitionen der Pharmaunternehmen in Forschung und Entwicklung angerechnet werden und langfristig in Investitionsanreize umgewandelt werden, regte Pfundner an. Bayerns Ministerpräsident Horst Seehofer zeigte sich für den Vorschlag offen. Er werde sich dafür in Berlin engagieren, erklärte Seehofer bei der Eröffnungsfeier.

Die neuen Produktions- und Laborräume sind in erster Linie für die biotechnologische Produktion von immundiagnosti- schen Tests vorgesehen. Dazu zählen bestimmte Krebsmarker, insbesondere für Brustkrebs und Tests für die Herz-KreislaufDiagnostik, sowie für Infektionskrankheiten wie Hepatitis und HIV. Darüber hinaus werden im DOC auch Forschungsreagenzien für den stark wachsenden Life Science Markt produziert. Roche beschäftigt in Penzberg mehr als 4.600 Mitarbeiter. In den vergangenen Jahren hat das Unternehmen seine Produktpalette für immundiagnostische Tests laufend ausgebaut. Allein 2009 gab es zwölf Markteinführungen auf globaler Ebene. Das DOC solle dazu beitragen, die Marktversorgung mit Immundiagnostika sicherzustellen und die weltweit führende Position von Roche in diesem Segment auszubauen, hieß es.

sto

Quelle: Pressekonferenz anlässlich der Einweihung des Diagnostics Operations Complex (DOC), Penzberg, 2. September 2010; Veranstalter: Roche Diagnostics

\title{
Tumorbedingte Durchbruchschmerzen Neue transmukosale Applikationen setzen Standard
}

Durchbruchschmerzen verlangen nach unverzüglicher Behandlung. "Die ideale Durchbruchschmerz-Medikation muss daher schnell und kurz wirken und sollte nach Möglichkeit nicht invasiv sein."

Diesen Forderungen entspricht nach Einschätzung von Hans Georg Kress, Wien, in optimaler Weise transmukosales Fentanyl in Form von Fentanyl-Buccaltabletten $\left(\right.$ Effentora $\left.^{\circledR}\right)$. Die Tabletten mit der neuartigen OraVescent ${ }^{\circledR}$-Technologie entfalten bereits ab der ersten Minute eine starke Wirkung und haben nach zehn Minuten bereits die volle analgetische Wirkung entfaltet. Wichtig sei, so Kress, dass in diesem Zusammenhang mit Irrtümern und Vorurteilen aufgeräumt werde. So habe sich noch immer nicht ausreichend die Erkenntnis durchgesetzt, dass bei Durchbruchschmerzen das Schmerzmaximum bereits nach wenigen Minuten erreicht sei und der Schmerz in der Regel nicht länger als 30 Minuten anhalte. Die Opioiddauermedikation kann diese Schmerzspitzen nicht auffangen. Daher wurden speziell für die Linderung von mittleren bis starken Durchbruchschmerzen bei erwachsenen Krebspatienten die neuen Buccaltabletten entwickelt. Für Kress stellen transmukosale Fentanyl-
Applikationsformen den neuen Standard in der Behandlung von Durchbruchschmerzen dar. Nicht nur in Studien [z.B. Slatkin NE et al. J Support Oncol 207; 5: 327-34], sondern auch in der alltäglichen Behandlung bei Patienten mit Durchbruchschmerzen habe sich erwiesen, dass die buccale Applikation effektiv und einfach anwendbar sei und so zur besseren Lebensqualität der $\mathrm{Pa}$ tienten beitrage, ergänzte Norbert Grießinger, Erlangen.

Quelle: Symposium im Rahmen des Deutschen Schmerzkongresses 2010, Mannheim, 7. Oktober 2010; Veranstalter: Cephalon 